\title{
Small-for-Gestational-Age Births in Pregnant Women with HIV, due to Severity of HIV Disease, Not Antiretroviral Therapy
}

\author{
Erika Aaron, ${ }^{1}$ Alexa Bonacquisti, ${ }^{2}$ Leny Mathew, ${ }^{3}$ Gregg Alleyne, ${ }^{4}$ \\ Laura P. Bamford, ${ }^{1}$ and Jennifer F. Culhane ${ }^{5}$ \\ ${ }^{1}$ Division of Infectious Diseases and HIV Medicine, Drexel University College of Medicine, 1427 Vine Street, 5th Floor, Philadelphia, \\ PA 19102, USA \\ ${ }^{2}$ Department of Psychology, Drexel University, 245 N 15th Street, MS 515, Philadelphia, PA 19102, USA \\ ${ }^{3}$ Department of Adolescent Medicine, Children's Hospital of Philadelphia, 3535 Market Street, Philadelphia, PA 19104, USA \\ ${ }^{4}$ Department of Obstetrics and Gynecology, Drexel University College of Medicine, 245 N 15th Street, MS 495, Philadelphia, PA 19102, \\ USA \\ ${ }^{5}$ Department of Pediatrics, University of Pennsylvania School of Medicine, 3535 Market Street, Philadelphia, PA 19104, USA
}

Correspondence should be addressed to Erika Aaron, eaaron@drexelmed.edu

Received 20 January 2012; Revised 20 April 2012; Accepted 22 April 2012

Academic Editor: Susan Cu-Uvin

Copyright ( $) 2012$ Erika Aaron et al. This is an open access article distributed under the Creative Commons Attribution License, which permits unrestricted use, distribution, and reproduction in any medium, provided the original work is properly cited.

\begin{abstract}
Objectives. To determine rate and factors associated with small-for-gestational-age (SGA) births to women with HIV. Methods. Prospective data were collected from 183 pregnant women with HIV in an urban HIV prenatal clinic, 2000-2011. An SGA birth was defined as less than the 10th or 3rd percentile of birth weight distribution based upon cut points developed using national vital record data. Bivariate analysis utilized chi-squared and $t$-tests, and multiple logistic regression analyses were used. Results. The prevalence of SGA was $31.2 \%$ at the 10 th and $12.6 \%$ at the 3 rd percentile. SGA at the 10 th (OR 2.77; 95\% CI, 1.28-5.97) and 3rd (OR 3.64; 95\% CI, 1.12-11.76) percentiles was associated with cigarette smoking. Women with CD4 count $>200$ cells/mm ${ }^{3}$ at the first prenatal visit were less likely to have an SGA birth at the 3rd percentile (OR 0.29; 95\% CI, 0.10-0.86). Women taking NNRTI were less likely to have an SGA infant at the 10th (OR 0.28; 95\% CI, 0.10-0.75) and 3rd (OR 0.16; 95\% CI, 0.03-0.91) percentiles compared to those women on PIs. Conclusions. In this cohort with high rates of SGA, severity of HIV disease, not ART, was associated with SGA births after adjusting for sociodemographic, medication, and disease severity.
\end{abstract}

\section{Introduction}

It is estimated that 8,700 women with HIV give birth annually in the United States [1]. Through improvements in early prenatal care initiation and the use of antiretroviral therapy (ART) during pregnancy, the rates of perinatal HIV transmission have decreased to as low as $1 \%$ to $2 \%$ in the developed world [2]. However, women with HIV are still at risk for adverse birth outcomes $[3,4]$. Research investigating birth outcomes of infants born to women with HIV has mainly concentrated on preterm birth (PTB; birth prior to 37 completed weeks of gestation) and low birth weight (LBW; birth at less than 2500 grams). Results from these studies are mixed with some studies showing an increased risk and others not showing this risk: PTB between 5\% and $20 \%$ and LBW between $10 \%$ and $20 \%$ [4]. It is unclear whether the increased risk is related to HIV infection and its consequences or to elevated prevalence rates of other risk factors for PTB and LBW among women with HIV [5].

We have chosen to focus on a measure combining both gestational age and birth weight: small for gestational age (SGA). Using cut points developed by Elo and Culhane, derived from national vital records data, each infant's status with regard to SGA was determined at both the 3rd and the 10th percentiles of gestational-age specific birth weight distributions. Infants born SGA experience higher rates of mortality and morbidity compared to infants born at appropriate weight for their GA [6]. Sequelae associated with SGA include both short- and long-term adverse outcomes, such as respiratory complications, hypotension, hypoglycemia, neurological impairment, type 2 diabetes mellitus, and cardiovascular complications, among others 
[6]. This definition of SGA is increasingly being used with most current studies investigating the risk factors for SGA, having used population centiles to define SGA [7]; however, few studies investigating outcomes in infants born to women with HIV have used the definition of SGA.

Published data conflict as to whether receipt of ART during pregnancy is associated with adverse pregnancy outcomes. This information is needed to inform healthcare providers for best practices and to improve the quality of life and health of women with HIV and their infants. The aim of this study was to determine the rate of SGA infants delivered to women with HIV and to assess contributing factors, with specific emphasis on the possible contribution of ART that may influence the likelihood that a woman with HIV will deliver an SGA infant.

\section{Methods}

A prospective cohort study was conducted among 183 pregnant women with HIV from January 2000 through January 2011 at Drexel University College of Medicine's Partnership Plus Clinic, an urban medical clinic in Philadelphia, PA specializing in prenatal HIV care. The study was approved by the Drexel University College of Medicine Institutional Review Board and conducted with the understanding and the consent of the participants. The study was designed to assess overall maternal health (biological and psychosocial) and infant health outcomes among pregnant women with HIV seeking care at this specialized comprehensive prenatal care clinic. Clinical care is delivered by an interdisciplinary team consisting of members of the Department of Infectious Diseases and the Department of Obstetrics and Gynecology. All women with HIV who were seen in this clinic over the identified period were approached at the time of their first prenatal visit. After informed consent was obtained from the women, data were collected through patient interviews and from medical chart review. Subjects were included in this study if they were HIV-infected, pregnant, and more than 17 years of age. Women who had an abortion or miscarriage, switched prenatal providers, or were incarcerated during the index pregnancy were excluded. Repeat pregnancies were not included, and only the first infant delivered in twin gestations (twin A) was included in these analyses.

2.1. Measures. Data were obtained through questionnaireguided interviews and medical chart abstraction. Interviews were conducted by one trained HIV clinician who saw all participants in this cohort; chart abstraction was completed by trained research assistants.

2.1.1. Demographic Data. Demographic data included age, race/ethnicity, education level (categorized as 8th grade; some high school; high school/GED; some college; 4-year college; master's degree or more), employment status, and marital status.

2.1.2. Health Behaviors. Health behaviors included smoking history and substance use history. Measures for smoking included ever smoking a cigarette as well as the prevalence of smoking at the time of the study. Crack cocaine, heroin, and marijuana use in lifetime as well as the prevalence at time of the study was used to create variables for illicit drug use.

2.1.3. Medical Data. Medical information pertaining to HIV that was abstracted from charts included HIV transmission route (categorized as unprotected sex with a man; intravenous drug use; blood transfusion; or other), date of ART initiation and regimen, CD4 T lymphocyte counts and HIV viral load measures at each medical visit, and year of HIV diagnosis. ART initiation was categorized as prepregnancy, first, second and at third trimester. Viral load and CD4 counts were used as continuous measures to evaluate trajectory over time. Viral load at the first and last visits were dichotomized at 1000 copies, and CD4 count at the first visit was dichotomized at 200 cells for final analysis.

Hospital obstetrical records were reviewed to collect data on birth outcomes. Variables included date of delivery, gestational age at delivery, gender, infant birth weight and length, and any neonatal or postpartum complications. Owing to the critical nature of gestational age in determining SGA status, we highlight this variable below. In addition, we discuss the construction of the main dependent variable SGA.

2.1.4. Gestational Age at Birth. Gestational age at birth was determined by the following criteria: patients who were dated by a sure LMP in first trimester were subsequently confirmed by the second trimester anatomy ultrasound. In those with unsure last menstrual period due to either history of irregular menstruation, the patient being a poor historian, or the pregnancy being conceived on hormonal contraception, the first trimester ultrasound was used as the dating tool. Those who presented to prenatal care after the first trimester with unsure last menstrual period were dated by a second or third trimester ultrasound depending on the gestational age at presentation to prenatal care.

2.2. SGA Birth. An SGA birth was defined as a newborn weighing less than the 10th percentile of gestational agespecific birth weight distribution based on the infant's sex and the mother's parity [8]. Specifically, to identify births as SGA we used reference curves developed based on vital statistics birth record data for singleton births born to US resident mothers in the 50 states and the District of Columbia in 1998, 1999, and 2000. Sex-specific reference curves for SGA cut points indicating the 10th percentile in the birth weight distribution for gestational ages 20-44 weeks were developed for first births and second or higher order births. In constructing the SGA cut points, births with extreme deviation from the mean birth weight for a given gestational week were excluded so that the established cut points were not influenced by extreme outliers in the data. We identified outliers by using standard procedures developed by Tukey whereby outliers were defined as births that fell outside the interquartile range of the birth weight distribution for a given gestational week. Based on these procedures approximately 
$1.5 \%$ of births were excluded in the construction of the standard. These reference curves (available from the authors upon request) update previously published standards [8]. In addition to the 10th percentile cut point, another SGA variable was created by dichotomizing at the 3rd percentile gestational age-specific birth weight distribution for a more stringent definition of SGA [9].

2.3. Statistical Analysis. For descriptive analysis, frequencies, percentages, prevalence rates, means, and standard deviations were calculated. Bivariate analyses were conducted to evaluate the association between SGA and the demographic, behavioral, and medical characteristics. Chi-square tests were used for comparing categorical variables. Fisher's exact test was utilized if the expected values in the cells were less than five. For comparison of maternal age, Student's $t$-test was used. Multiple logistic regression was then used to analyze the risk of SGA, adjusting for covariates and potential confounders. Variables that were significant at the 0.25 level in the bivariate analyses were included in the logistic regression model. Using a 0.25 level of significance as an inclusion criterion for the regression model allows us to consider a wider pool of potentially important covariates, some of which may eventually be significant in the multivariate model. It has been noted that traditional significance levels of 0.05 can fail to identify variables known to be important in models [10-12]. Since the use of nonnucleoside reverse transcriptase inhibitor (NNRTI) and protease inhibitor (PI) medications is correlated, separate models were run to evaluate the associations on SGA with the same covariates. A generalized linear model was fitted to the CD4 count data to evaluate differences in rate of change of CD4 over time between the SGA groups. The generalized linear model used a gamma distribution and log link function along with robust standard errors and adjusted for the clustering of the data by participant. The Gamma distribution was used due to the skewed nature of the CD4 data. Time in months since entry into care was used as the time variable, and an interaction term of time with the SGA group was used to assess differences in rate of change of $\mathrm{CD} 4$ over time. A similar model was used to evaluate differences in rate of change of viral load over time.

All data were coded and entered in SPSS version 18 (IBM, Armonk, NY); Stata 11.2 (StataCorp, College Station, TX) was used for all analyses.

\section{Results}

Baseline characteristics of the sample are presented in Table 1. Out of 221 pregnancies the number of discreet women included in this analysis was 183 . Our final sample of 183 women was reached because of the following exclusions: 20 repeat deliveries; 2 twins; 5 births with incomplete delivery information; 4 abortions or miscarriages; 3 changed prenatal providers; and 4 incarcerations during the index pregnancy. The cohort was mostly African American (74.7\%, $n=136)$ and single $(86.7 \%, n=157)$ with a mean age of 28.0 years $(S D=6.2)$. Educational attainment was low
TABLe 1: Sociodemographic, behavioral, and medical characteristics of sample.

\begin{tabular}{|c|c|}
\hline Variable & $N(\%)$ \\
\hline Age-mean (SD) & $28.0(6.2)$ \\
\hline Race-African American & $136(74.7)$ \\
\hline Race-all other & $46(25.3)$ \\
\hline Married & $24(13.3)$ \\
\hline Single & $157(86.7)$ \\
\hline Education-less than high school & $36(19.7)$ \\
\hline Education—high school/some high school & $80(43.7)$ \\
\hline Education-college/some college & $19(10.4)$ \\
\hline Education-unknown & $48(26.2)$ \\
\hline Parity-no children & $55(30.9)$ \\
\hline Parity -1 or more children & $123(69.1)$ \\
\hline Ever smoked & $93(50.8)$ \\
\hline Currently smoking & $70(38.3)$ \\
\hline Ever used illicit drugs & $81(44.3)$ \\
\hline Currently using illicit drugs & $33(18.0)$ \\
\hline First viral load $>1000$ & $101(55.2)$ \\
\hline Last viral load > 1000 & $27(14.8)$ \\
\hline First CD4 > 200 & $134(73.2)$ \\
\hline Started medications in pregnancy & $137(74.9)$ \\
\hline ART started: prepregnancy & $46(25.1)$ \\
\hline ART started: first trimester & $26(14.2)$ \\
\hline ART started: second trimester & $90(49.2)$ \\
\hline ART started: third trimester & $21(11.5)$ \\
\hline NNRTI & $39(21.3)$ \\
\hline PI & $117(63.9)$ \\
\hline SGA, 10th percentile & $57(31.2)$ \\
\hline SGA, 3rd percentile & $23(12.6)$ \\
\hline Gestational age at care entry-mean (SD) & $14.6(7.5)$ \\
\hline Gestational age at delivery-mean (SD) & $38.0(2.1)$ \\
\hline Infant birth weight (grams) — mean (SD) & $2900.0(523.4)$ \\
\hline Low birth weight & $31(16.9)$ \\
\hline Preterm birth & $34(18.6)$ \\
\hline
\end{tabular}

ART, antiretroviral therapy; NNRTI, nonnucleoside reverse transcriptase inhibitor; PI, protease inhibitor; SD, standard deviation; SGA, small for gestational age.

(high school or less; $63.4 \%, n=116$ ). A total of $69.1 \%$ $(n=123)$ of the women reported at least one birth prior to this pregnancy; $38.3 \%(n=70)$ reported smoking during this pregnancy; $44.3 \%(n=81)$ reported a history of ever using illicit drugs (majority of illicit drug use in this cohort was cocaine), and $18.0 \%(n=33)$ reported using illicit drugs during the current pregnancy. The mean GA of the infant at the time the mother entered care was 14.6 weeks $(\mathrm{SD}=$ $7.5)$; the mean GA at outcome was 38 weeks $(S D=2.1)$. There were no significant differences of the GA at entry into prenatal care by SGA at the 10th percentile ( 14.8 weeks; SD = 7.4, $P=0.7029)$ or SGA at the 3 rd percentile (13.2 weeks; $\mathrm{SD}=3.7, P=0.9630$; Table 2 ). The average duration of time since HIV diagnosis for the cohort was 4.3 years $(\mathrm{SD}=4.3)$. The incidence of preeclampsia was $13.6 \%(n=21 / 150)$. 
TABLE 2: Socio-demographic, behavioral, and medical characteristics, by SGA at 10th percentile and SGA at 3rd percentile.

\begin{tabular}{|c|c|c|c|c|c|c|}
\hline \multirow{2}{*}{ Variable } & \multicolumn{3}{|c|}{ 10th percentile } & \multicolumn{3}{|c|}{ 3rd percentile } \\
\hline & $\begin{array}{c}\text { SGA: no } \\
n=126(\%)\end{array}$ & $\begin{array}{l}\text { SGA: yes } \\
n=57(\%)\end{array}$ & $P$ value & $\begin{array}{c}\text { SGA: no } \\
n=160(\%)\end{array}$ & $\begin{array}{l}\text { SGA: yes } \\
n=23(\%)\end{array}$ & $P$ value \\
\hline Age-mean $(\mathrm{SD})$ & $27.7(5.8)$ & $28.7(7.0)$ & 0.411 & $27.9(6.1)$ & $29(6.7)$ & 0.337 \\
\hline Race-African American & $89(71.2)$ & $47(82.5)$ & \multirow{2}{*}{0.105} & $119(74.8)$ & $17(73.9)$ & \multirow{2}{*}{0.924} \\
\hline Race- all other & $36(28.8)$ & $10(17.5)$ & & $40(25.2)$ & $6(26.1)$ & \\
\hline Married & $17(13.6)$ & $7(12.5)$ & \multirow{2}{*}{0.840} & $21(13.2)$ & $3(13.6)$ & \multirow{2}{*}{0.956} \\
\hline Single & $108(87.5)$ & $49(87.5)$ & & $138(86.8)$ & $19(86.4)$ & \\
\hline Education-less than high school & $24(19.1)$ & $12(21.1)$ & \multirow{3}{*}{0.650} & $27(16.9)$ & $9(39.1)$ & \multirow[b]{2}{*}{0.028} \\
\hline $\begin{array}{l}\text { Education-high school/some } \\
\text { high school }\end{array}$ & $58(46.0)$ & $22(38.6)$ & & $73(45.6)$ & $7(30.4)$ & \\
\hline Education—college/some college & $11(8.7)$ & $8(14.0)$ & & $15(9.4)$ & $4(17.4)$ & \\
\hline Education-unknown & $33(26.2)$ & $15(26.3)$ & \multirow{3}{*}{0.893} & $45(28.1)$ & $3(13.0)$ & \multirow{3}{*}{0.666} \\
\hline Parity-no children & $37(30.6)$ & $18(31.6)$ & & $47(30.3)$ & $8(34.8)$ & \\
\hline Parity -1 or more children & $84(69.4)$ & $49(68.4)$ & & $108(69.7)$ & $15(65.2)$ & \\
\hline Never smoked & $62(49.2)$ & $18(31.6)$ & \multirow{3}{*}{0.057} & $74(46.3)$ & $6(26.1)$ & \multirow{3}{*}{0.153} \\
\hline Ever smoked & $59(46.8)$ & $34(59.7)$ & & $77(48.1)$ & $16(69.6)$ & \\
\hline Ever smoked—unknown & $5(4.0)$ & $5(8.8)$ & & $9(5.6)$ & $1(4.4)$ & \\
\hline Not currently smoking & $75(59.5)$ & $27(47.4)$ & \multirow{3}{*}{0.250} & $93(58.1)$ & $9(39.1)$ & \multirow{3}{*}{0.056} \\
\hline Currently smoking & $45(35.7)$ & $27(43.9)$ & & $57(35.6)$ & $13(56.5)$ & \\
\hline Current smoking—unknown & $6(4.8)$ & $5(8.8)$ & & $10(6.3)$ & $1(4.4)$ & \\
\hline Never used illicit drugs & $71(56.4)$ & $26(45.6)$ & \multirow{3}{*}{0.396} & $85(53.1)$ & $12(52.2)$ & \multirow{3}{*}{0.671} \\
\hline Ever used illicit drugs & $52(41.3)$ & $29(50.9)$ & & $70(43.8)$ & $11(47.8)$ & \\
\hline Ever used illicit drugs—unknown & $3(2.4)$ & $2(3.5)$ & & $5(3.1)$ & $0(0)$ & \\
\hline Not currently using illicit drugs & $97(76.9)$ & $44(77.2)$ & \multirow{3}{*}{0.984} & $122(77.3)$ & $19(82.6)$ & \multirow{3}{*}{0.495} \\
\hline Currently using illicit drugs & $23(18.3)$ & $10(17.5)$ & & $29(18.1)$ & $4(17.4)$ & \\
\hline $\begin{array}{l}\text { Currently using illicit } \\
\text { drugs_-unknown }\end{array}$ & $6(4.8)$ & $3(5.3)$ & & $9(5.6)$ & $0(0)$ & \\
\hline First viral load $>1000$ & $63(50.0)$ & $38(66.7)$ & 0.036 & $87(54.4)$ & $14(60.9)$ & 0.558 \\
\hline Last viral load $>1000$ & $17(13.5)$ & $10(17.5)$ & 0.474 & $22(13.8)$ & $5(21.8)$ & 0.312 \\
\hline First CD4 >200 & $94(74.6)$ & $40(70.2)$ & 0.531 & $122(76.3)$ & $12(52.2)$ & 0.015 \\
\hline Started medications in pregnancy & $91(72.2)$ & $46(80.7)$ & 0.221 & $116(72.5)$ & $21(91.3)$ & 0.052 \\
\hline ART started: prepregnancy & $35(27.8)$ & $11(19.3)$ & \multirow{4}{*}{0.532} & $44(27.5)$ & $2(8.7)$ & \multirow{4}{*}{0.069} \\
\hline ART started: first trimester & $19(15.1)$ & $7(12.3)$ & & $23(14.4)$ & $3(13.0)$ & \\
\hline ART started: second trimester & $58(46.0)$ & $32(56.1)$ & & $73(45.6)$ & $17(73.9)$ & \\
\hline ART started: third trimester & $14(11.1)$ & $7(12.3)$ & & $20(12.5)$ & $1(4.4)$ & \\
\hline NNRTI & $32(25.4)$ & $7(12.3)$ & 0.045 & $37(23.1)$ & $2(8.7)$ & 0.114 \\
\hline PI & $78(61.9)$ & $39(68.4)$ & 0.395 & $100(62.5)$ & $17(73.9)$ & 0.287 \\
\hline $\begin{array}{l}\text { Gestational age at care } \\
\text { entry-mean (SD) }\end{array}$ & $14.6(7.6)$ & $14.9(7.4)$ & 0.7029 & $14.8(7.9)$ & $13.2(3.6)$ & 0.9630 \\
\hline $\begin{array}{l}\text { Gestational age at delivery_-mean } \\
\text { (SD) }\end{array}$ & $38.2(1.9)$ & $37.6(2.6)$ & 0.3071 & $38.1(2.0)$ & $37.9(3.0)$ & 0.7364 \\
\hline $\begin{array}{l}\text { Infant birth weight (grams) } \\
\text { - mean (SD) }\end{array}$ & $3111.1(392.2)$ & $2434.2(475.1)$ & $<0.001$ & $2980.2(479.9)$ & $2344.1(481.6)$ & $<0.001$ \\
\hline Low birth weight & $5(4.0)$ & $26(45.6)$ & $<0.001$ & $17(10.6)$ & $14(60.9)$ & $<0.001$ \\
\hline Preterm birth & $19(15.1)$ & $15(26.3)$ & 0.070 & $31(19.4)$ & $3(13.0)$ & 0.465 \\
\hline
\end{tabular}

ART, antiretroviral therapy; NNRTI, nonnucleoside reverse transcriptase inhibitor; PI, protease inhibitor; SD, standard deviation; SGA, small for gestational age. 
Two perinatal transmissions occurred in this cohort; neither were SGA. The first infant was 37 weeks gestation, 2952 grams. Maternal factors included ART of NRTI and NNRTI (nevirapine); CD4 count of 20; and viral load at the time of delivery, 98,500. The second infant was 42.2 week-gestation, 3430.3 grams. Maternal factors included ART of NRTI and PI combination (lopinavir/ritonavir); CD4 count of 626, viral load at time of delivery, 1000. It was suspected that transmission occurred during breastfeeding immediately following delivery.

CD4 T lymphocyte counts and HIV viral loads were recorded throughout pregnancy and at the time of delivery. The majority of women had CD4 counts greater than 200 copies $/ \mathrm{mm}^{3}(n=134,73.2 \%)$; $55.2 \%(n=101)$ had viral loads greater than 1000 copies $/ \mathrm{mL}$ at entry into prenatal care and $72.1 \%(n=132)$ were diagnosed with HIV prior to the index pregnancy. Disease severity as measured by CD4 at entry into care, and length of time of HIV diagnosis did not vary significantly between the women taking NNRTIs and those taking PIs. The number of women with CD4 counts greater than 200 and taking PIs (146) was 105 (71.9\%) whereas the number of those taking NNRTIs (43) was 30 $(60.8 \%), P=0.78$. Average duration of time since HIV diagnosis for those taking PIs was 5.08 years $(\mathrm{SD}=4.7)$ compared with 4.72 years $(\mathrm{SD}=4.3 ; P=0.884)$ for those taking NNRTIs.

All women were taking ART during the pregnancy; 100\% $(n=183)$ had nucleoside reverse transcriptase inhibitors (NRTIs) as part of their background regimen, with $14.8 \%$ $(n=27)$ taking NRTIs alone; $21.3 \%(n=39)$ were taking nonnucleoside reverse transcriptase inhibitors (NNRTIs), and $63.9 \%(n=117)$ were taking protease inhibitors (PIs). There were $11(40.7 \%)$ women who were taking NRTI only and whose infants were SGA at the 10th percentile, as compared to all other women (on either a PI and NNRTI) whose infants were SGA at the 10th percentile $(46,29.5 \%$ $P=0.244)$. Four (14.8\%) women were taking NRTI only whose infants were SGA at the 3rd percentile as compared to all others who were SGA at the 3rd percentile (19, 12.2\% $P=0.703)$. Therefore, those women taking NRTI only did not have a higher rate of SGA at the 10th or 3rd percentiles.

Of the women taking NNRTIs, $1.1 \%(n=2)$ were taking efavirenz, $16.9 \%(n=31)$ were taking nevirapine, and $3.3 \%(n=6)$ were taking etravirine. The following PIs were used in this cohort: amprenavir $(2.7 \%, n=5)$; atazanavir $(15.8 \%, n=29)$; darunavir $(1.1 \%, n=2)$; lopinavir/ritonavir $(20.8 \%, n=38)$; fosamprenavir $(1.1 \%$, $n=2)$; nelfinavir $(23 \%, n=42)$; and ritonavir $(15.8 \%$, $n=29)$. No participants were taking indinavir, raltegravir, or saquinavir. The majority of this sample $(n=137 ; 74.9 \%)$ was not taking ART prior to the index pregnancy and started the HIV medication regimen during the prenatal period.

We found that ritonavir did not have the adverse outcome of an SGA infant as others have reported [13, 14]. There were 67 women on ritonavir-boosted regimens compared to 116 who were not exposed to ritonavir. A total of $22(32.8 \%)$ women were taking ritonavir who also had SGA infants at the 10th versus 35 (30.2\%) women not taking ritonavir who had SGA infants at the 10th $(P=0.708)$. A total of $8(11.9 \%)$ women taking ritonavir also had SGA at the 3 rd versus $15(12.9 \%)$ women not taking ritonavir with SGA infants at the 3 rd percentile $(P=0.846)$. The overall prevalence of SGA was high with $31.2 \%$ at the 10 th percentile and $12.6 \%$ at the $3 \mathrm{rd}$ percentile. In bivariate analyses at the 10th percentile, newborns were less likely to be SGA if their mother was taking NNRTI medication compared to those newborns whose mothers were not taking NNRTI medication $(12.3 \%$ versus $25.4 \% ; P=0.045)$. Newborns were more likely to be SGA if their mother's first viral load was $>1000$ copies $/ \mathrm{mL}(50.0 \%$ versus $66.7 \% ; P=0.036$. There were no significant differences in the rates of SGA in bivariate analyses for the following factors: the first available CD4 value dichotomized at 200 cells $/ \mathrm{mm}^{3}$; the rate of change of CD4 and viral load during the prenatal period; starting ART during pregnancy compared to starting ART prior to pregnancy or even starting ART later in pregnancy; taking a PI; length of time with HIV diagnosis; and viral load measurements at entry into the prenatal period and every trimester thereafter. There were no significant differences in those infants delivered preterm who were at the 10th percentile $(n=15,26.3 \%)$ compared to the non-SGA infants ( $n=19,15.1 \% ; P=0.070)$. As expected, the birth weight for those with SGA at the 10th percentile was significantly less than those without SGA $(P<0.001)$. A higher percentage of the women with SGA infants had ever smoked cigarettes $(P=0.057)$, though smoking at the time of the study $(P=$ 0.250 ) was not statistically significant for an SGA infant at the 10th percentile (Table 2).

The same comparisons were reevaluated using SGA at the 3 rd percentile where the rate was shown to be $12.6 \%$ (Table 2). Education status was significantly associated with SGA at the 3rd percentile with a higher percentage of women with SGA infants having less than a high school education $(P=0.028)$. Current smoking status was close to significant $(P=0.056)$. CD4 counts $>200$ cells $/ \mathrm{mm}^{3}$ at the first prenatal visit were significantly associated with a decreased risk of SGA at the 3rd percentile $(P=0.015)$. All other factors were not significantly associated with SGA at the 3rd percentile including HIV viral load measurements at entry into the prenatal period and every trimester thereafter, choice of ART (either NNRTI $[P=0.114]$ or PI $[P=0.287]$ ), initiation of ART during the index pregnancy compared to those who started ART prior to the index pregnancy $(P=0.052)$, or starting ART in the third trimester. There were no significant differences in those infants delivered preterm at the $3 \mathrm{rd}$ percentile $(n=3,13 \%)$ as compared to the non-SGA infants ( $n=31,19.4 \% ; P=0.465)$. As expected, the birth weight for those with SGA at the 3rd percentiles was significantly less than those without SGA $(P<0.001)$.

Cesarean delivery was the most prevalent mode of delivery for all infants, regardless of SGA status. Among the women for whom the mode of delivery was known $(n=193$, $87 \%$ ), $54 \%$ of the deliveries were cesarean births. SGA was not associated with an increased risk of cesarean delivery. The two SGA groups had comparable distribution of vaginal and cesarean deliveries. The modes of delivery of those infants at the 10th percentile were vaginal, $42.1 \%(n=24)$; cesarean, $54.4 \%(n=31)$; and unknown $3.5 \%(n=2)$; for those at 
the 3rd percentile, vaginal deliveries were $39.1 \%(n=9)$ and cesarean deliveries were $60.9 \%(n=14)$.

The results of the logistic regression models are presented in Table 3. In the adjusted model for SGA at the 10th percentile, cigarette smoking was significantly associated with a higher likelihood of an SGA birth (OR 2.77; 95\% CI, 1.28-5.97). Interestingly, even after adjustment, women taking NNRTI medications were significantly less likely to have an SGA birth at the 10th percentile (OR 0.28; 95\% CI, 0.10-0.75) compared to those treated with other HIV regimens.

In the adjusted model for SGA at the 3rd percentile, current cigarette smoking was associated with a higher likelihood of an SGA birth (OR 3.64; 95\% CI, 1.12-11.76). Those who had a CD4 value greater than 200 cells $/ \mathrm{mm}^{3}$ at the first clinic visit were significantly less likely to have an SGA infant (OR 0.29, 95\% CI, 0.10-0.86). As at the 10th percentile, after adjustment, women taking NNRTI medications were significantly less likely to have an SGA birth at the 3rd percentile (OR 0.16; 95\% CI, 0.03-0.91) compared to those treated with other HIV regimens.

The rates of SGA in this cohort remained exceptionally elevated even when compared to a rate of 16\% SGA births defined as less than the 10th percentile observed in a similar group of HIV-negative non-Hispanic black women from Philadelphia, PA. The comparison dataset was from a community-based study examining maternal stress and maternal and infant health and health-related behaviors [15]. The women were recruited from a consortium of eight health centers run by the Philadelphia Department of Public Health. The sample used for comparison is a subsample of 3990 on whom SGA data were available.

When we formally compare the 183 to the 3990 , we find the following differences: the HIV-infected cohort is significantly older $(M=28.0 ; \mathrm{SD}=6.2)$ compared to the HIV negative comparison $(M=24.1 ; \mathrm{SD}=5.7$; $P<.001)$. The HIV-infected cohort has a significantly higher percentage of women who were African American $(74.7 \%$ versus $67.1 \% \quad P=0.03)$, and single $(86.7 \%, 75.7 \% \quad P=$ 0.001 ), and HIV-infected women are more likely to have greater parity (more than 1 child: $69.1 \%$ versus $55.7 \% P=$ $0.001)$ and higher rates of ever having smoked $(50.8 \%$ versus $35.1 \%, P=0.001)$. In our sample of HIV-infected women the risk factors associated with SGA are more prevalent; however, when accounted for in the multivariate model, none except smoking was statistically significant.

\section{Discussion}

The present study sought to determine the rate of SGA deliveries among HIV-infected pregnant women, and to assess contributing factors that may influence the likelihood that an HIV-infected woman will deliver an SGA infant. A high rate of SGA births was observed in this sample: $31.2 \%$ of the sample delivered an SGA infant at the 10th percentile and $12.6 \%$ of the sample delivered an SGA infant at the 3rd percentile. These rates remain exceptionally elevated even when compared to an SGA rate of $16 \%$, defined using the same cut points at the 10th percentile, and observed in a sociodemographically at-risk cohort of HIV-negative nonHispanic black women from Philadelphia, PA [8]. Further, the rate of SGA, defined at the 3rd percentile, in this cohort of HIV-positive women was $12.6 \%$ as compared to the national average of $3 \%$.

In this prospective observational study, several factors emerged as potential contributors to this increased rate. The severity of HIV disease, but not the ART regimen, was associated with a significantly increased rate of SGA at the 3 rd percentile. Infants born to mothers with a CD4 $>200$ were $70 \%$ less likely to be SGA at the 3 rd percentile compared to infants born to mothers with CD4 $\leq 200$ (95\% CI, 0.10 0.86). However, CD4 was not significantly associated with increased risk of SGA at the 10th percentile. The length of time with a diagnosis of HIV did not affect SGA outcome in this cohort.

Another factor that could explain the high rate of SGA infants is that this cohort has sociodemographic and behavior characteristics that have been noted to contribute to SGA outcomes. We compared this cohort to a dataset of 3990 women who were recruited from a consortium of eight health centers run by the Philadelphia Department of Public Health that targeted vulnerable populations. The HIV group had more of the risk factors associated with SGA infants, but after accounting for these risk factors in the multivariate model, only smoking was significantly associated with SGA outcomes. After adjusting for maternal sociodemographic and behavioral characteristics, women taking NNRTIs were less likely to have SGA infants in both at the 10th percentile (OR 0.28; 95\% CI, $0.10-0.75 ; P=0.045$ ) and at the 3rd percentile (OR 0.16; 95\% CI, 0.03-0.91), though the 3rd percentile was not significant $(P=0.114)$. Published data are conflicting as to whether receipt of ART during pregnancy is associated with adverse pregnancy outcomes. A large US single-center study found a 1.8 -fold increase in preterm delivery among women who received PIs as compared to those on a non-PI containing regimen. After adjustment for possible confounders including disease severity, only combination therapy with a PI was associated with risk for preterm delivery compared to any other regimen (95\% CI, 1.1-3.0) [16]. However, those receiving PIs had more advanced disease in this cohort. The European Collaborative Study and the Swiss Mother and Child HIV Cohort Study [17] report that even with adjustment for CD4 count and injection drug use an odds ratio for prematurity of 2.60 (95\% CI, 1.43-4.75) and 1.82 (95\% CI, 1.13-2.92) for infants exposed to ART with or without a PI, respectively, was compared to no treatment. In contrast, in a meta-analysis of seven prospective clinical studies comparing women on antenatal ART with those not on ART (1990-1998), no increase in the rate of preterm birth or low birth weight was observed in women taking ART [18]. Other studies also report no significant associations between use of ART by class or category and adverse pregnancy outcome [19-22].

Associations between timing of the initiation of ART and risk of preterm births have been reported. One large cohort study showed women receiving ART initiated before their pregnancy were twice as likely to deliver prematurely as those starting therapy during the third trimester [17]. In 
TABLE 3: Logistic regressions predicting 10th percentile and 3rd percentile.

\begin{tabular}{lcccc}
\hline Variable & \multicolumn{2}{c}{ 10th percentile } & \multicolumn{2}{c}{ 3rd percentile } \\
Confidence interval
\end{tabular}

${ }^{*}$ Run as a separate model with the same covariate.

NNRTI, nonnucleoside reverse transcriptase inhibitor; PI, protease inhibitor.

an updated report from this same cohort, the risk of delivery before 34 weeks of gestation was increased by 2.5 -fold for those starting ART during pregnancy and 4.4-fold for those entering pregnancy on ART as compared to those women not on ART [23]. Powis et al. [24] found that PI-based highly active antiretroviral therapy (HAART) initiated in the third trimester of pregnancy was associated with a 2-fold higher odds of a preterm delivery compared with triple NRTI-based HAART begun at the same time. In our study, those who started ART after prenatal care initiation compared to those who were on ART prior to pregnancy had a trend toward SGA at the 3 rd percentile $(P=0.052)$, but not at the 10 th percentile.

Mode of delivery was not associated with an increased risk of SGA at either the 10th or the 3rd percentile. The effect of cigarette smoking during the index pregnancy at the 10th percentile (OR 2.77; 95\% CI, 1.28-5.97) and the 3rd percentile (OR 3.64; 95\% CI, 1.12-11.76) was significantly associated with a higher likelihood of an SGA birth. However, other established risk factors for SGA, such as gestational and chronic hypertension, and preeclampsia were not evaluated in this cohort due to inadequate data collection on these variables. Of the 150 women who had available data on preeclampsia, we found higher rates of preeclampsia $(13.6 \%$, $n=21)$, compared to the national average of $2-3 \%$ of all pregnancies (5-7\% in nulliparous women) [25]. This finding may be due to the provision of regular antenatal care in this cohort, including management of HIV status, sexually transmitted infections, hypertension, and diabetes which could contribute to the lack of association with these important potential risks for SGA [7].
In attempting to decipher why this cohort had exceptionally high levels of SGA infants, it is possible that exposure to HIV has an independent association with increased risk of SGA as a result of immune activation. Ongoing immune activation is associated with poor recovery of CD4+ T cells during early and long-term ART and might contribute to the pathogenesis of non-AIDS-related HIV diseases, such as atherosclerotic vascular disease and non-AIDS-related cancers [26-28]. Studies investigating the effect of HIV and pregnancy on the immune system have shown that even with low levels of viremia, HIV-infected women at delivery showed an immunologic profile different from that of both healthy non-HIV-infected women in the puerperium and nonpregnant women, with lower CD4 T lymphocytes and higher CD8 T lymphocytes, high levels of CD38 expression, but low CD56 expression on CD8+ T cells and low natural killer cell numbers $[29,30]$. Fiore et al. [31] have proposed ART immunomodulation as a potential mechanism triggering preterm deliveries. This group proposed that immune reconstitution with resultant cytokine shifts may underlie the association. In the current study, the rate of change in CD4 cells between women experiencing SGA deliveries and those experiencing non-SGA deliveries was not significantly different. Further investigation into this potential mechanism is required; possibly a larger sample will be able to demonstrate a more robust association.

Our study had several limitations. Due to the limited number of participants, the study was underpowered to detect an association with less prevalent risk factors. Due to the length of data collection over 10 years, the ART regimens had greater variations than if this study were completed over 
a shorter period. Additionally, we did not collect information on body mass index, which is an indicator of poor fetal outcomes. Future studies should include this variable. A strength of this study is the use of the SGA outcome, because it is a more sensitive measure of adverse outcomes than is preterm delivery or low gestational weight. Few studies have explored SGA as an outcome in this population [32]. We were able to use robust data for SGA definition based upon curves developed from vital statistics data for singleton births born to US resident mothers in the 50 states and the District of Columbia in 1998, 1999, and 2000. Additionally, we used a comparison dataset of 3990 women who were recruited from a consortium of eight health centers run by the Philadelphia Department of Public Health for whom SGA data were available. A strength of this study was the ability to use these data sets, which helped to quantify SGA in this small but important population. Additionally, this is a prospective study, thus eliminating the inconsistencies of a retrospective study.

\section{Conclusions}

In this prospective longitudinal cohort study of women with HIV, we found there was an increase in SGA births compared to a similar HIV negative population: $31.2 \%$ as compared to $16 \%$. However, we found no association between increased risk of SGA birth and class and category of ART. Interestingly, NNRTIs had a protective effect for the risk of SGA infants. ART is a critical component in the prevention of perinatal transmission in both the developed and developing world and offers proven benefits to maternal and infant health. Many uncertainties remain regarding potential adverse effects of taking ART during pregnancy. The association of SGA in this study was likely due to severity of HIV disease, not ART. Future research to better understand the pathophysiology of SGA in pregnant women with HIV is warranted to better characterize the risk factors associated with delivering an SGA infant.

\section{References}

[1] S. K. Whitmore, X. Zhang, A. W. Taylor, and J. M. Blair, "Estimated number of infants born to HIV-infected women in the United States and five dependent areas, 2006," Journal of Acquired Immune Deficiency Syndromes, vol. 57, no. 3, pp. 218-222, 2011.

[2] Centers for Disease Control and Prevention, "Mother-toChild (Perinatal) HIV Transmission and Prevention," 2007, http://www.cdc.gov/hiv/topics/perinatal/resources/factsheets /perinatal.htm.

[3] J. R. Cocroft, W. W. Hauck, L. Cosler, and B. J. Turner, "The effect of ethnicity and maternal birthplace on small-forgestational-age deliveries to HIV-infected women," Journal of Urban Health, vol. 79, no. 1, pp. 147-160, 2002.

[4] J. Mirpuri and L. Jain, "Issues of prematurity and HIV infection," Clinics in Perinatology, vol. 37, no. 4, pp. 887-905, 2010.

[5] P. Traisathit, J. Y. Mary, S. Le Cour et al., "Risk factors of preterm delivery in HIV-infected pregnant women receiving zidovudine for the prevention of perinatal HIV," Journal of
Obstetrics and Gynaecology Research, vol. 35, no. 2, pp. 225233, 2009.

[6] P. Saenger, P. Czernichow, I. Hughes, and E. O. Reiter, "Small for gestational age: short stature and beyond," Endocrine Reviews, vol. 28, no. 2, pp. 219-251, 2007.

[7] L. McCowan and R. P. Horgan, "Risk factors for small for gestational age infants," Best Practice and Research, vol. 23, no. 6, pp. 779-793, 2009.

[8] I. T. Elo, J. F. Culhane, I. V. Kohler et al., "Neighbourhood deprivation and small-for-gestational-age term births in the United States," Paediatric and Perinatal Epidemiology, vol. 23, no. 1, pp. 87-96, 2009.

[9] J. Gardosi, "New definition of small for gestational age based on fetal growth potential," Hormone Research, vol. 65, no. 3, pp. 15-18, 2006.

[10] Z. Bursac, C. H. Gauss, D. K. Williams, and D. W. Hosmer, "Purposeful selection of variables in logistic regression," Source Code for Biology and Medicine, vol. 3, article 17, 2008.

[11] R. B. Bendel and A. A. Afifi, "Comparison of stopping rules in forward "stepwise" regression," Journal of the American Statistical Association, vol. 72, pp. 46-53, 1977.

[12] R. M. Mickey and S. Greenland, "The impact of confounder selection criteria on effect estimation," American Journal of Epidemiology, vol. 129, no. 1, pp. 125-137, 1989.

[13] J. Sibiude, J. Warszawski, R. Tubiana et al., "Premature delivery in HIV-infected women starting protease inhibitor therapy during pregnancy: role of the ritonavir boost?" Clinical Infectious Diseases, vol. 54, no. 9, pp. 1348-1360, 2012.

[14] F. Kakkar, V. Lamarre, H. Soudeyns et al., "Use of boosted protease inhibitor in pregnancy and risk of prematurity among HIV-exposed uninfected infants: Canada," in Proceedings of the 19th Conference on Retroviruses and Opportunistic Infections, Poster \#1026, Seattle Wash, USA, March 2012.

[15] E. F. Dennis, D. A. Webb, S. A. Lorch, L. Mathew, J. R. Bloch, and J. F. Culhane, "Subjective social status and maternal health in a low income urban population," Maternal and Child Health Journal, vol. 16, no. 4, pp. 834-843, 2011.

[16] A. M. Cotter, A. G. Garcia, M. L. Duthely, B. Luke, and M. J. O'Sullivan, "Is antiretroviral therapy during pregnancy associated with an increased risk of preterm delivery, low birth weight, or stillbirth?" Journal of Infectious Diseases, vol. 193, no. 9, pp. 1195-1201, 2006.

[17] M.-L. Newell, C. Aebi, M. Battegay et al., "Combination antiretroviral therapy and duration of pregnancy," AIDS, vol. 14, no. 18, pp. 2913-2920, 2000.

[18] R. E. Tuomala, D. E. Shapiro, L. M. Mofenson et al., "Antiretroviral therapy during pregnancy and the risk of an adverse outcome," New England Journal of Medicine, vol. 346, no. 24, pp. 1863-1870, 2002.

[19] K. Patel, D. E. Shapiro, S. B. Brogly et al., "Prenatal protease inhibitor use and risk of preterm birth among HIV-infected women initiating antiretroviral drugs during pregnancy," Journal of Infectious Diseases, vol. 201, no. 7, pp. 1035-1044, 2010.

[20] R. E. Tuomala, H. Watts, D. Li et al., "Improved obstetric outcomes and few maternal toxicities are associated with antiretroviral therapy, including highly active antiretroviral therapy during pregnancy," Journal of Acquired Immune Deficiency Syndromes, vol. 38, no. 4, pp. 449-473, 2005.

[21] E. G. Szyld, E. M. Warley, L. Freimanis et al., "Maternal antiretroviral drugs during pregnancy and infant low birth weight and preterm birth," AIDS, vol. 20, no. 18, pp. 23452353, 2006. 
[22] A. P. Kourtis, C. H. Schmid, D. J. Jamieson, and J. Lau, "Use of antiretroviral therapy in pregnant HIV-infected women and the risk of premature delivery: a meta-analysis," AIDS, vol. 21, no. 5, pp. 607-615, 2007.

[23] C. Thorne, D. Patel, and M. L. Newell, "Increased risk of adverse pregnancy outcomes in HIV-infected women treated with highly active antiretroviral therapy in Europe," AIDS, vol. 18, no. 17, pp. 2337-2339, 2004.

[24] K. M. Powis, D. Kitch, A. Ogwu et al., "Increased risk of preterm delivery among HIV-infected women randomized to protease versus nucleoside reverse transcriptase inhibitorbased HAART during pregnancy," Journal of Infectious Diseases, vol. 204, no. 14, pp. 506-514, 2011.

[25] Royal College of Obstetricians and Gynaecologists, "Preeclampsia-study group consensus statement," 2003, http://www.rcog.org.uk/womens-health/clinical-guidance/pre -eclampsia-study-group-consensus-statement.

[26] A. N. Phillips, J. Neaton, and J. D. Lundgren, "The role of HIV in serious diseases other than AIDS," AIDS, vol. 22, no. 18, pp. 2409-2418, 2008.

[27] M. A. French, M. S. King, J. M. Tschampa, B. A. Da Silva, and A. L. Landay, "Serum immune activation markers are persistently increased in patients with HIV infection after 6 years of antiretroviral therapy despite suppression of viral replication and reconstitution of CD4+ T Cells," Journal of Infectious Diseases, vol. 200, no. 8, pp. 1212-1215, 2009.

[28] P. W. Hunt, J. N. Martin, E. Sinclair et al., "T cell activation is associated with lower $\mathrm{CD} 4+\mathrm{T}$ cell gains in human immunodeficiency vires-infected patients with sustained viral suppression during antiretroviral therapy," Journal of Infectious Diseases, vol. 187, no. 10, pp. 1534-1543, 2003.

[29] J. M. Denney, E. L. Nelson, P. D. Wadhwa et al., "Longitudinal modulation of immune system cytokine profile during pregnancy," Cytokine, vol. 53, no. 2, pp. 170-177, 2011.

[30] H. H. M. Truong, M. S. Sim, M. Dillon et al., "Correlation of immune activation during late pregnancy and early postpartum with increases in plasma HIV RNA, CD4/CD8 T cells, and serum activation markers," Clinical and Vaccine Immunology, vol. 17, no. 12, pp. 2024-2028, 2010.

[31] S. Fiore, E. Ferrazzi, M. L. Newell, D. Trabattoni, and M. Clerici, "Protease inhibitor-associated increased risk of preterm delivery is an immunological complication of therapy," Journal of Infectious Diseases, vol. 195, no. 6, pp. 914-916, 2007.

[32] N. Parekh, H. Ribaudo, S. Souda et al., "Risk factors for very preterm delivery and delivery of very-small-for-gestationalage infants among HIV-exposed and HIV-unexposed infants in Botswana," International Journal of Gynecology and Obstetrics, vol. 115, no. 1, pp. 20-25, 2011. 


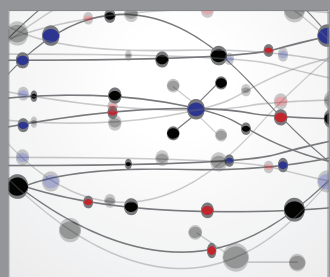

The Scientific World Journal
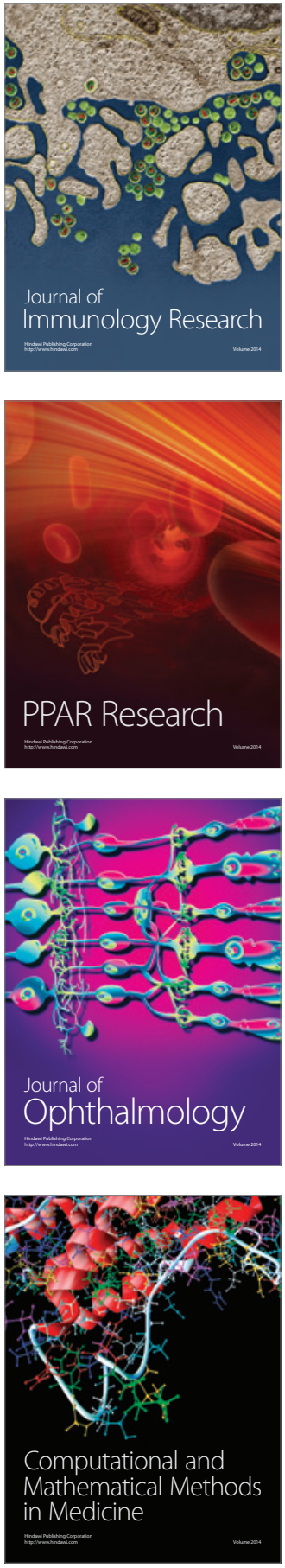

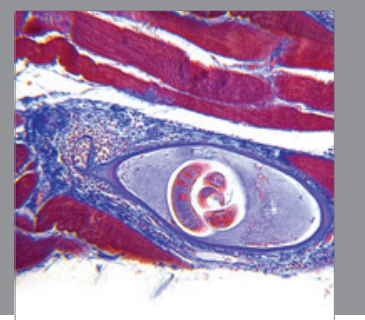

Gastroenterology

Research and Practice
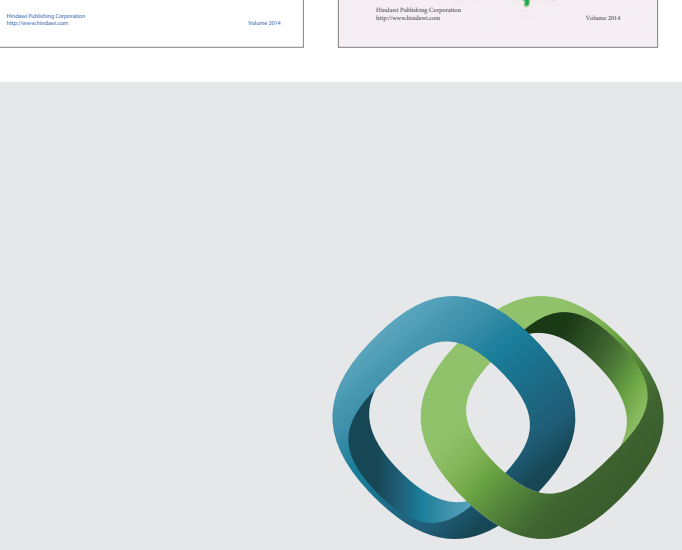

\section{Hindawi}

Submit your manuscripts at

http://www.hindawi.com
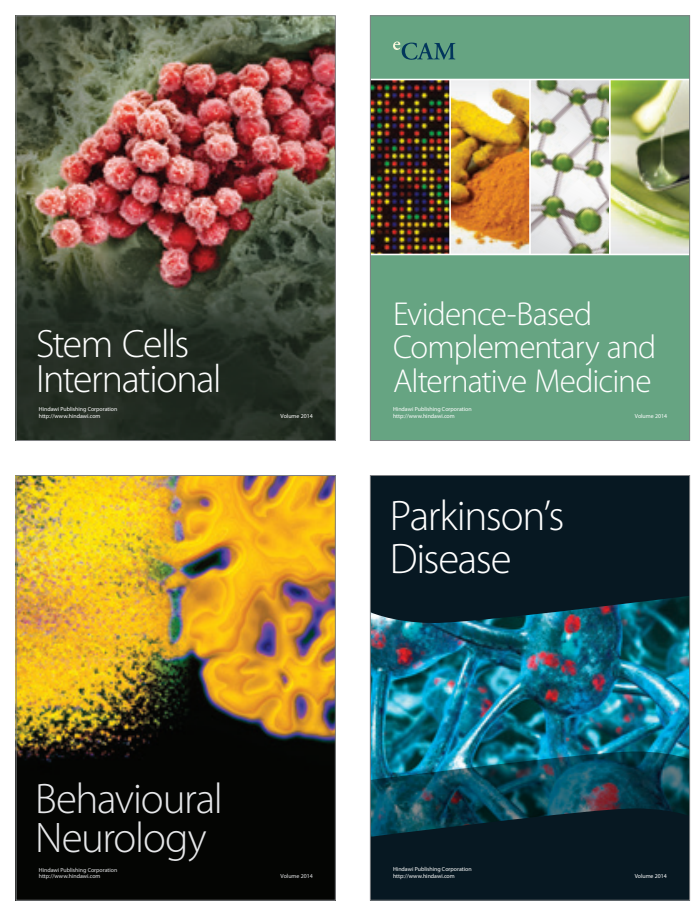

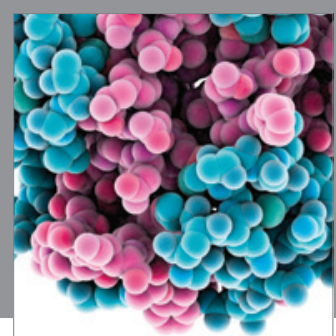

Journal of
Diabetes Research

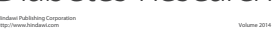

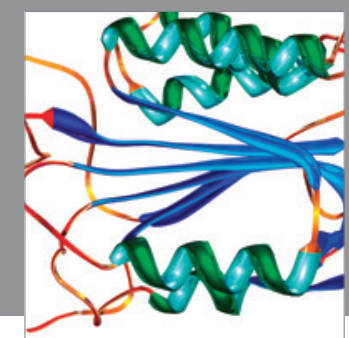

Disease Markers
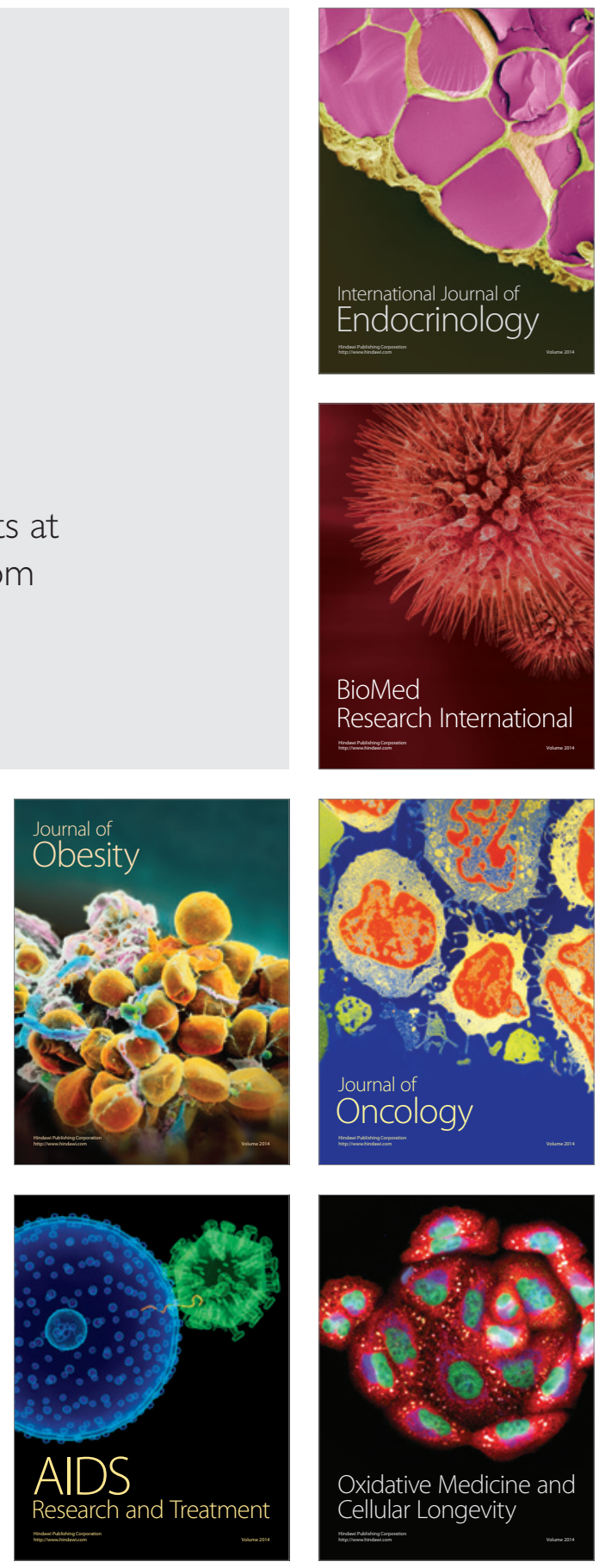